\title{
Tuning the Hydrogen Evolution Performance of Metallic 2D Tantalum Disulfide by Interfacial Engineering
}

Qiangmin Yu, ${ }^{\dagger}$ Yuting Luo, ${ }^{\dagger}$ Siyao Qiu, ${ }^{\dagger}$ Qinye Li,${ }^{\S}$ Zhengyang Cai, ${ }^{\dagger}$ Zhiyuan Zhang, ${ }^{\dagger}$ Jiaman Liu, ${ }^{\dagger}$ Chenghua Sun, ${ }^{z, *}$ and Bilu Liu ${ }^{\dagger, *}$

†Shenzhen Geim Graphene Center (SGC), Tsinghua-Berkeley Shenzhen Institute (TBSI) \& Tsinghua Shenzhen International Graduate School (TSIGS), Tsinghua University, Shenzhen 518055, P. R. China

tScience \& Technology Innovation Institute, Dongguan University of Technology, Dongguan 523808, China

$\S$ School of Chemical Engineering, Monash University, Clayton, Victoria 3800, Australia

${ }^{\text {Z}}$ Department of Chemistry and Biotechnology, and Center for Translational Atomaterials, Swinburne University of Technology, Hawthorn, Victoria 3122, Australia

Corresponding authors: bilu.liu@sz.tsinghua.edu.cn; chenghuasun@swin.edu.au 

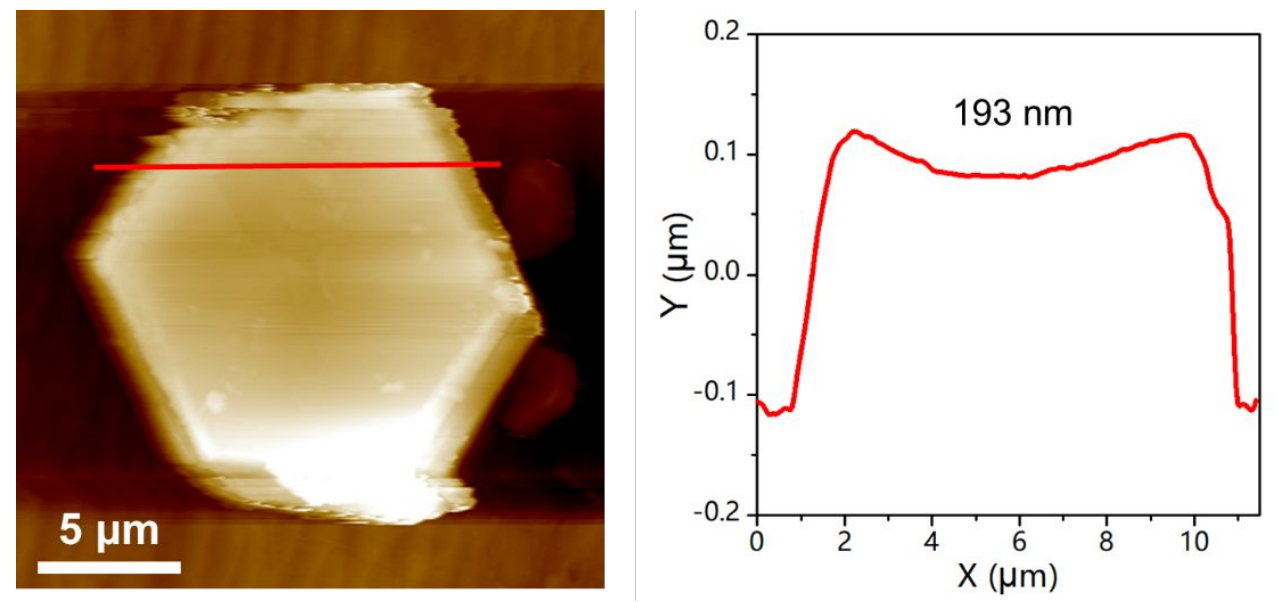

Figure S1. AFM image and corresponding height profile of $\mathrm{TaS}_{2}$. The samples were grown on $\mathrm{Au}$ substrate with a growth time of $60 \mathrm{~min}$, and transferred onto $\mathrm{Si} / \mathrm{SiO}_{2}$ substrate for AFM measurements.

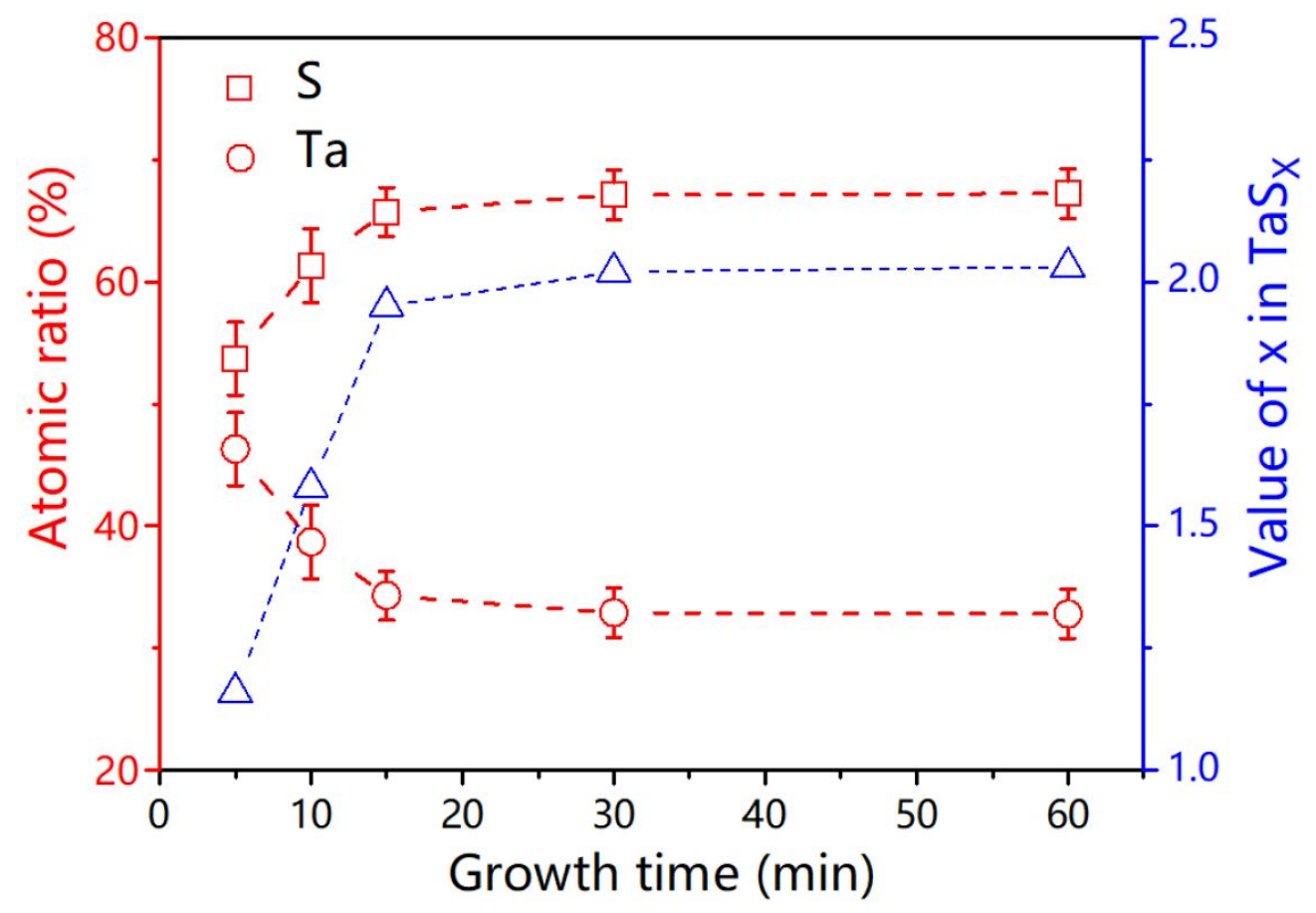

Figure S2. Ta/S atomic ratio (left y-axis) of CVD grown $\mathrm{TaS}_{2}$ on $\mathrm{Au}$ foil and the value of $\mathrm{x}$ in $\mathrm{TaS}_{\mathrm{x}}$ (right $\mathrm{y}$-axis) with different growth times from 5 to $60 \mathrm{~min}$. 

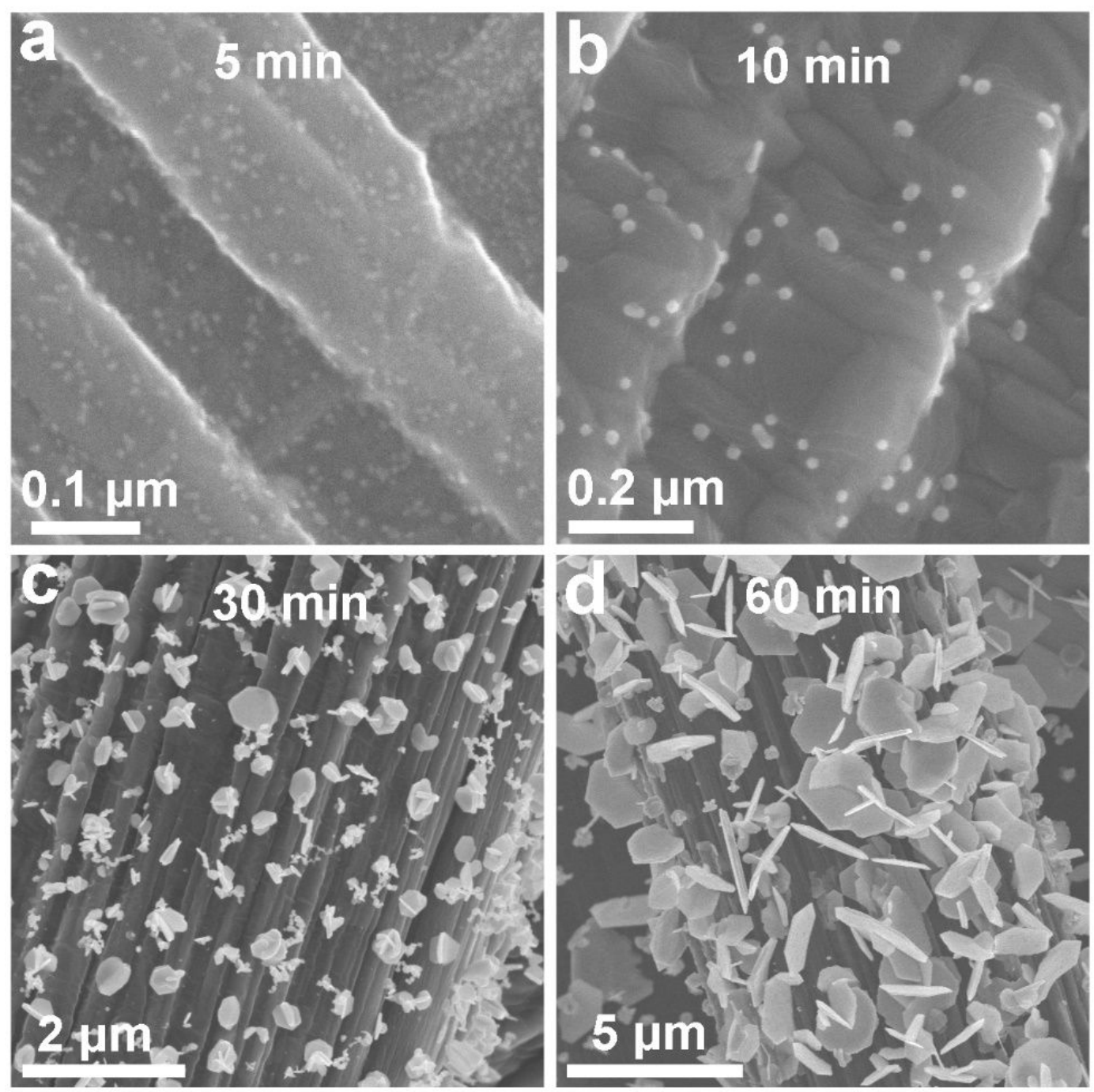

Figure S3. SEM images of $\mathrm{TaS}_{2}$ grown on a carbon fiber (CF) substrate with different growth times. (a) 5min, (b) $10 \mathrm{~min}$, (c) $30 \mathrm{~min}$, and (d) $60 \mathrm{~min}$. 

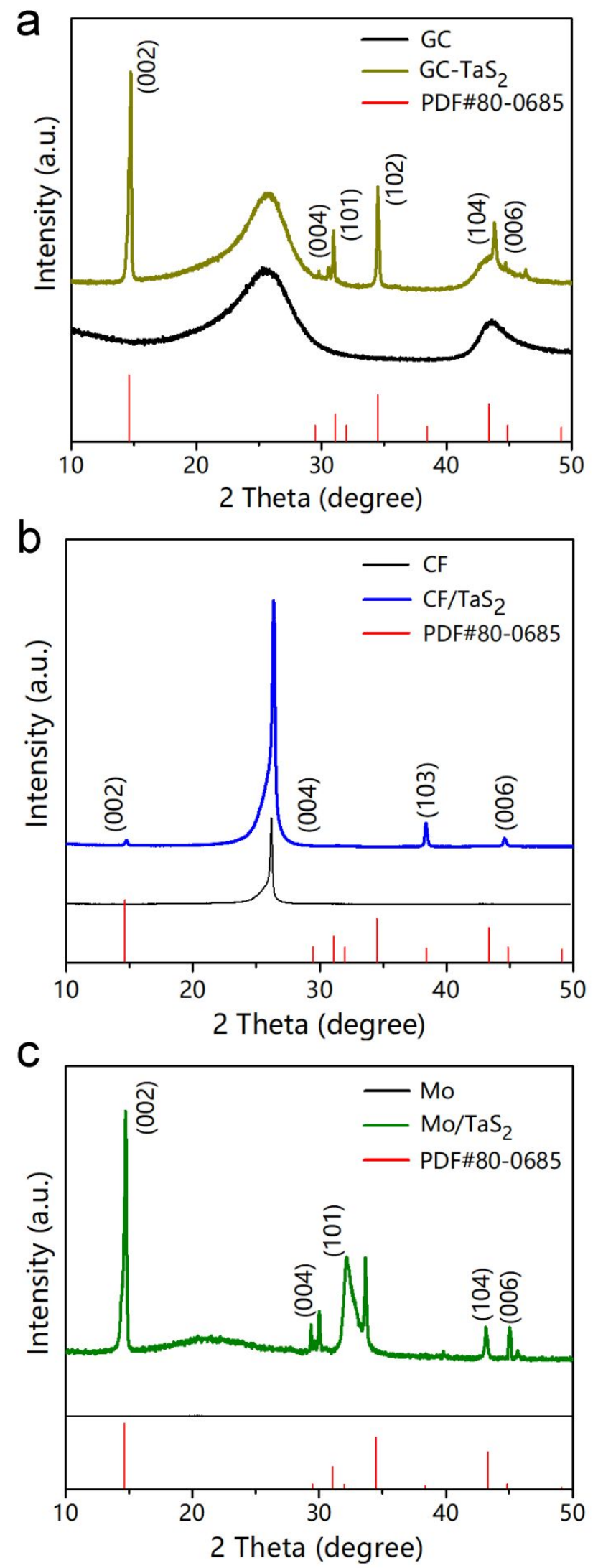

Figure S4. XRD patterns of (a) $\mathrm{GC} / \mathrm{TaS}_{2}$, (b) $\mathrm{CF} / \mathrm{TaS}_{2}$, and (c) $\mathrm{Mo} / \mathrm{TaS}_{2}$ showing the growth of $2 \mathrm{H}$ phase $\mathrm{TaS}_{2}$ on these substrates, the same as on the Au substrate. 

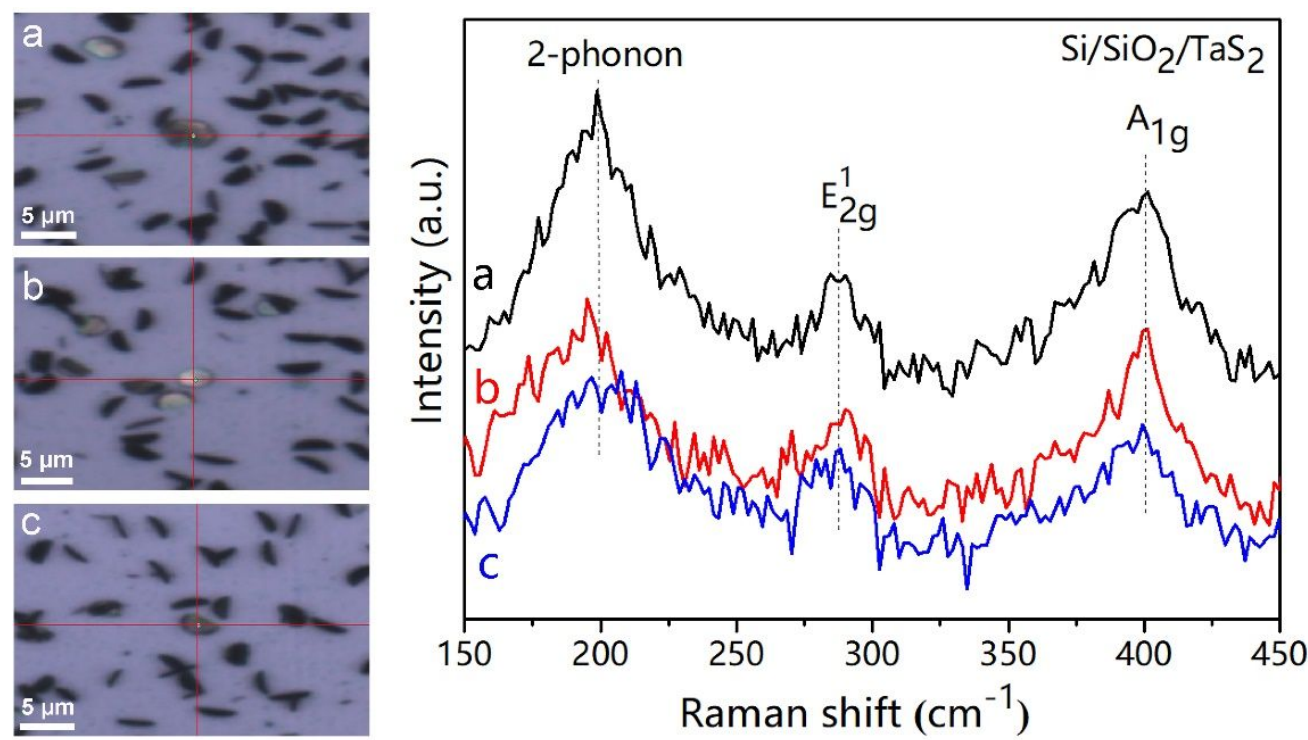

Figure S5. Optical microscopy images and Raman spectra of $\mathrm{TaS}_{2}$ showing its $2 \mathrm{H}$ phase structure. We transferred the $\mathrm{TaS}_{2}$ samples from $\mathrm{Au}$ foil by bath ultrasonic method. After sonication, the $\mathrm{TaS}_{2}$ samples were dispersed in ethanol and then dropped onto $\mathrm{Si} / \mathrm{SiO}_{2}$ substrate for Raman measurement.

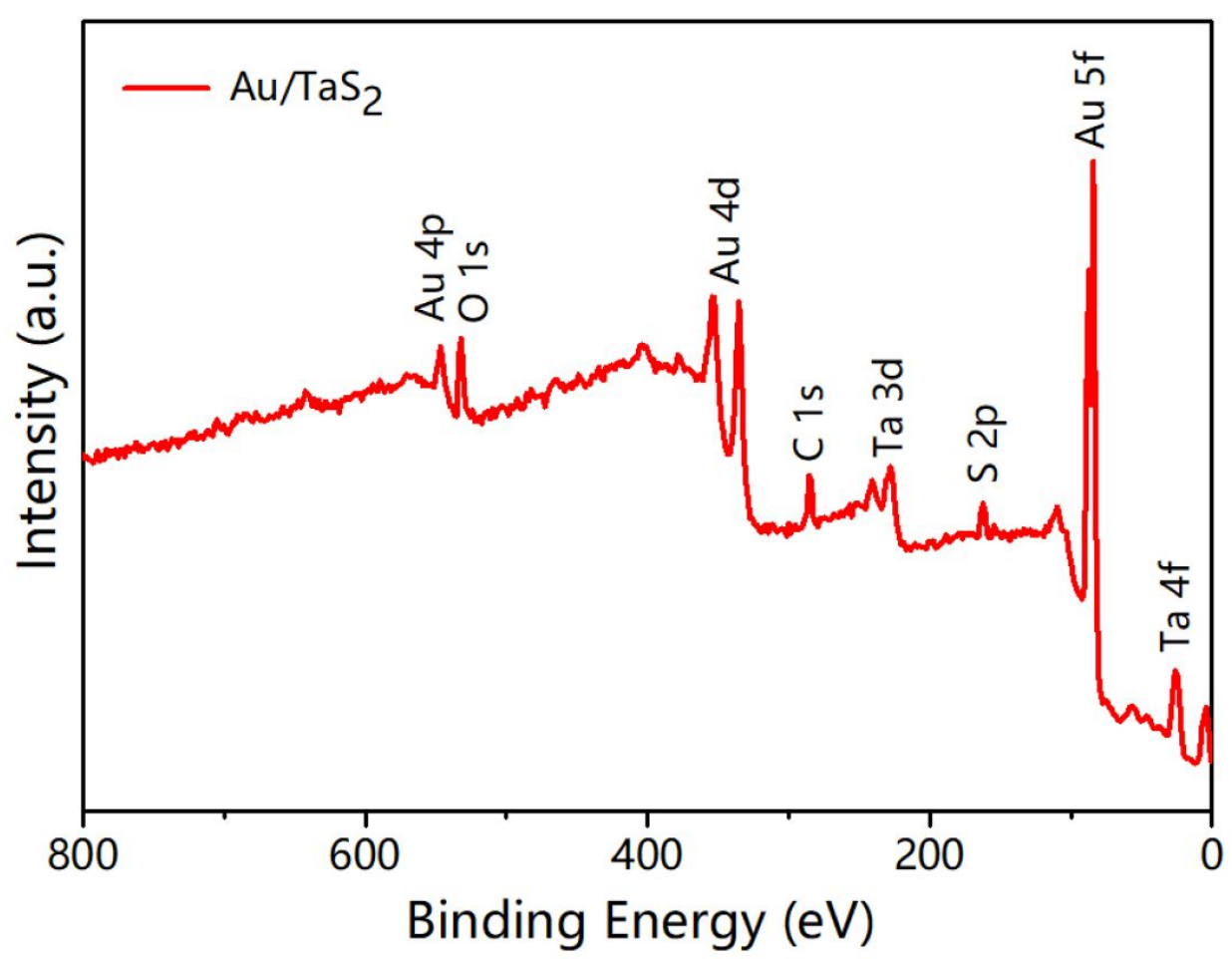

Figure S6. Survey XPS spectrum of $\mathrm{Au} / \mathrm{TaS}_{2}$, showing the existence of $\mathrm{Au}, \mathrm{Ta}$, and $\mathrm{S}$. 

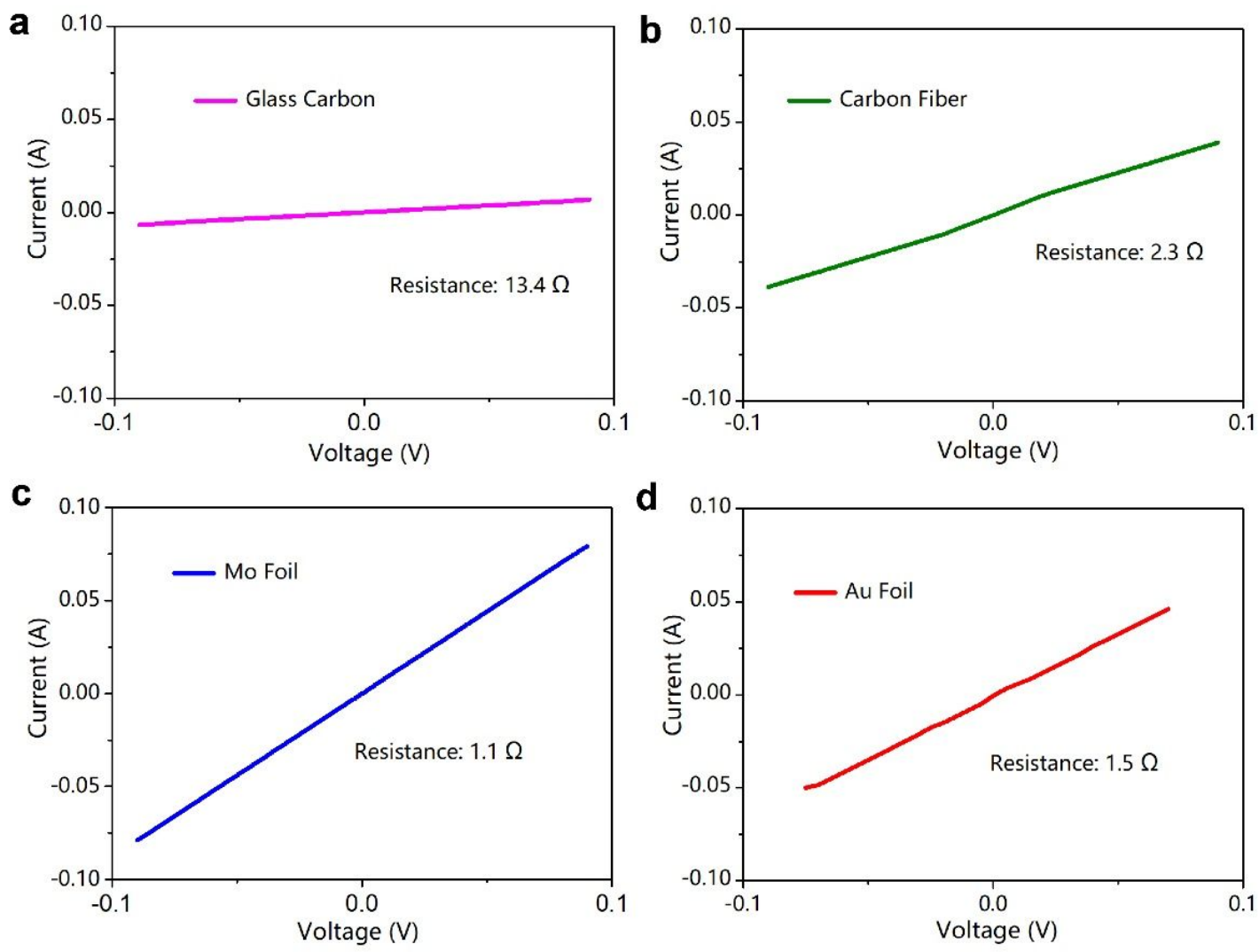

Figure S7. Two-terminal measurement of I-V curves of the different substrates. (a) GC;

(b) CF; (c) Mo foil; (d) Au foil. 

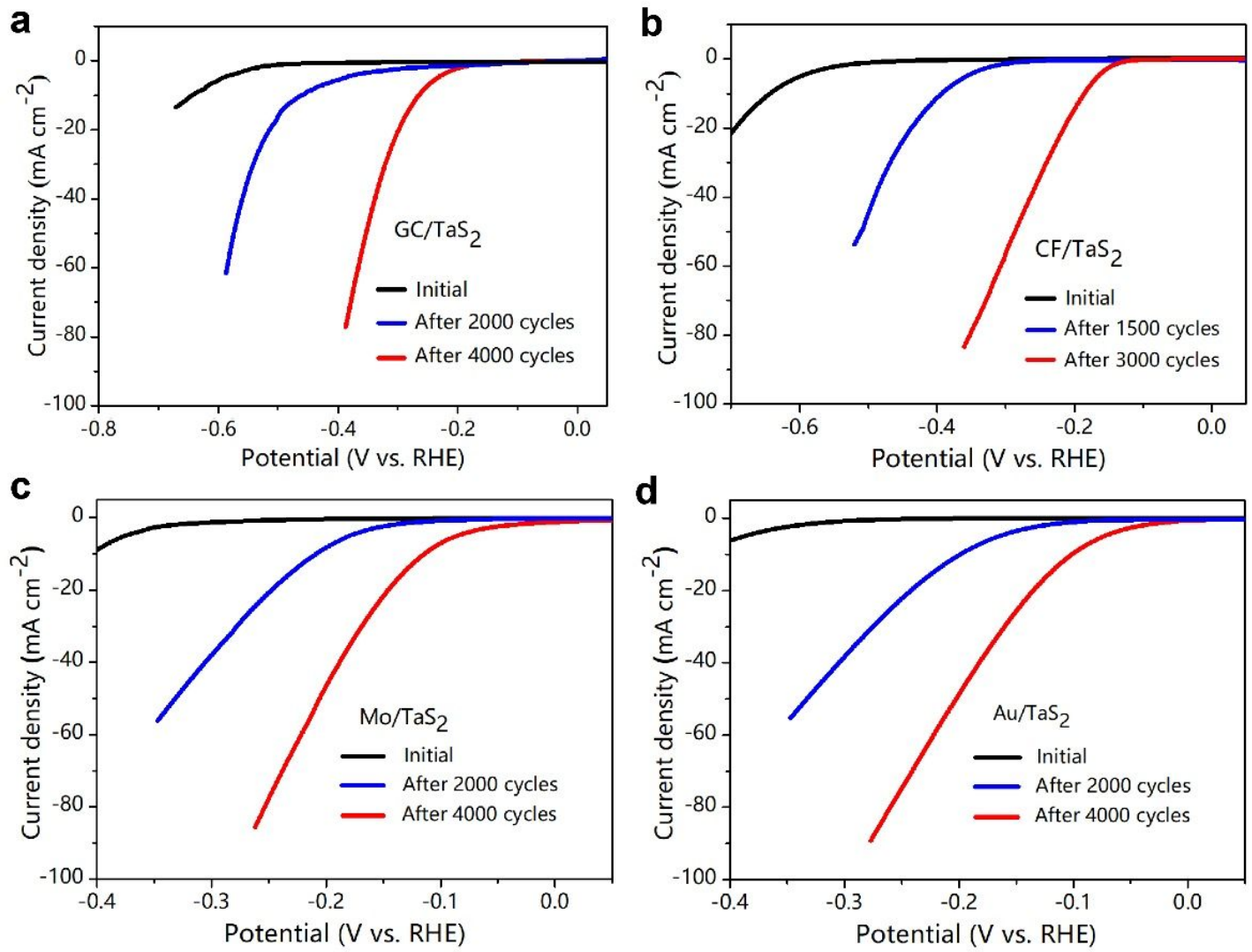

Figure S8. Polarization curves of the different catalysts recorded after cycling. The initial polarization curve was recorded after 20 cycles. (a) $\mathrm{GC} / \mathrm{TaS}_{2}$; (b) $\mathrm{CF} / \mathrm{TaS}_{2}$; (c) $\mathrm{Mo} / \mathrm{TaS}_{2}$; (d) $\mathrm{Au} / \mathrm{TaS}_{2}$. The samples show a gradually improved performance during cycling, consistent with recent reports. ${ }^{1,2}$ 


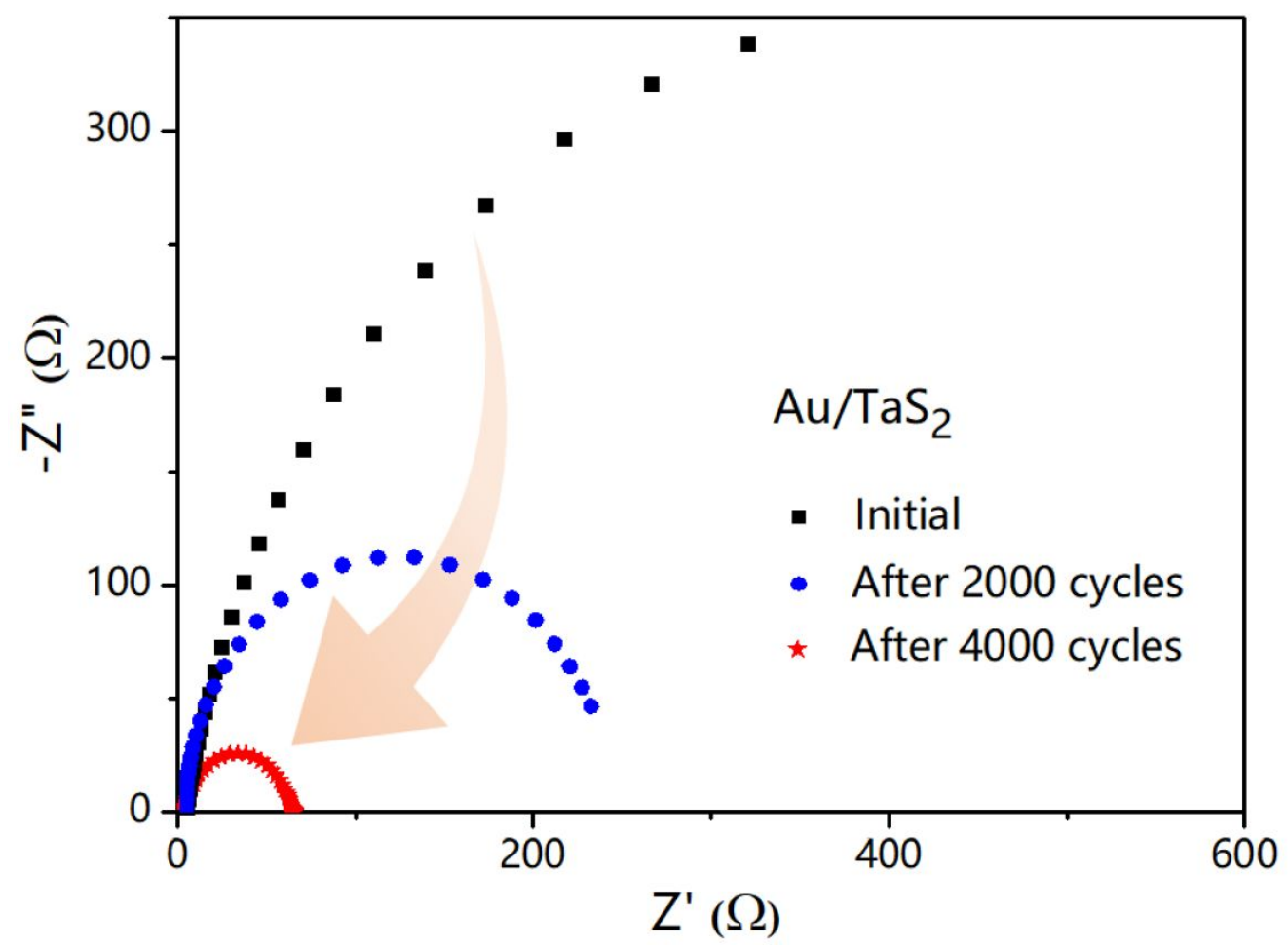

Figure S9. Electrochemical impedance measurements of $\mathrm{Au} / \mathrm{TaS}_{2}$ after different numbers of CV cycles.
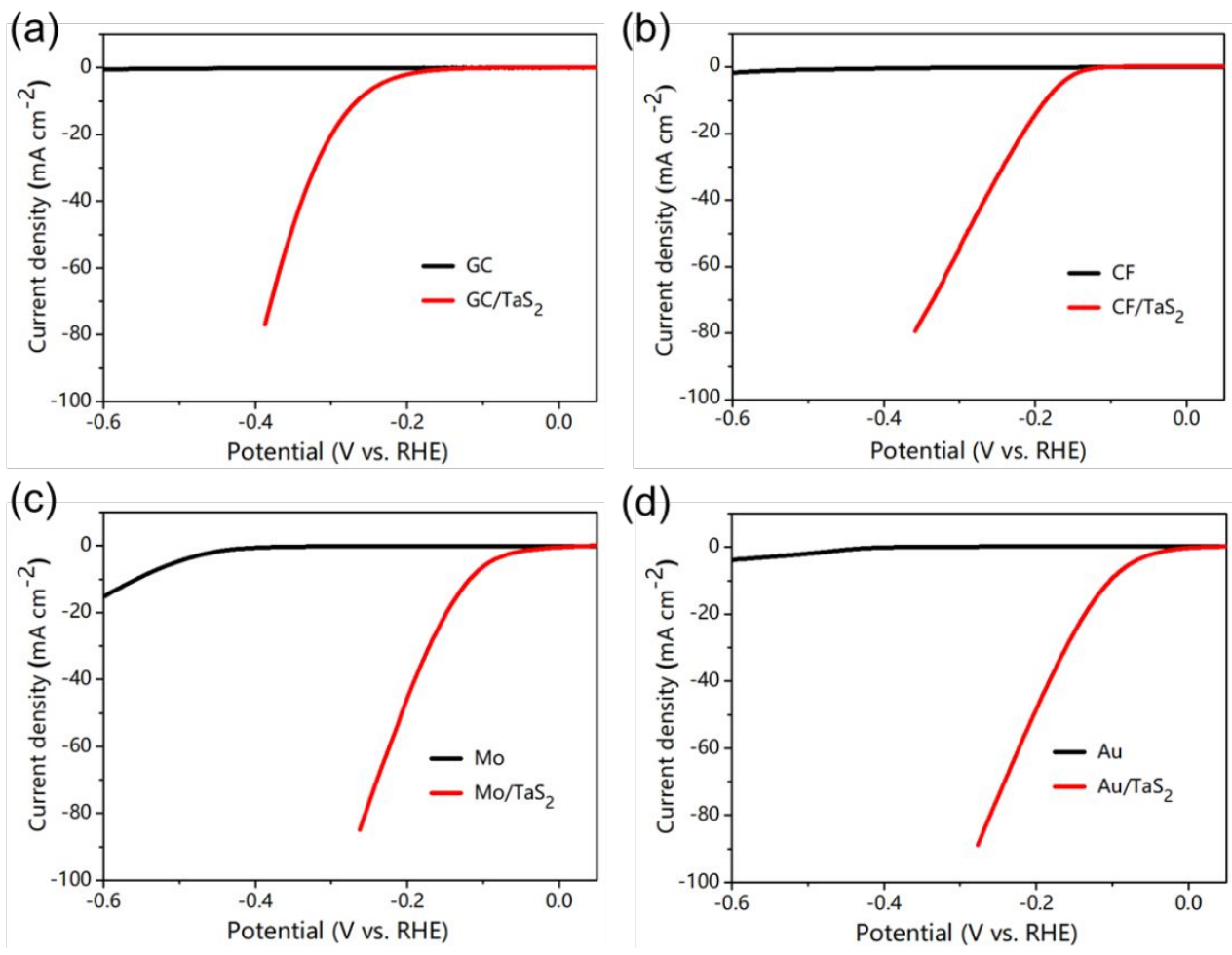
Figure S10. HER polarization curves of different substrates, with (red) and without (black) $\mathrm{TaS}_{2}$ grown, including GC, CF, Mo and Au foils. The results confirm that the substrates have negligible contribution to the HER performance.

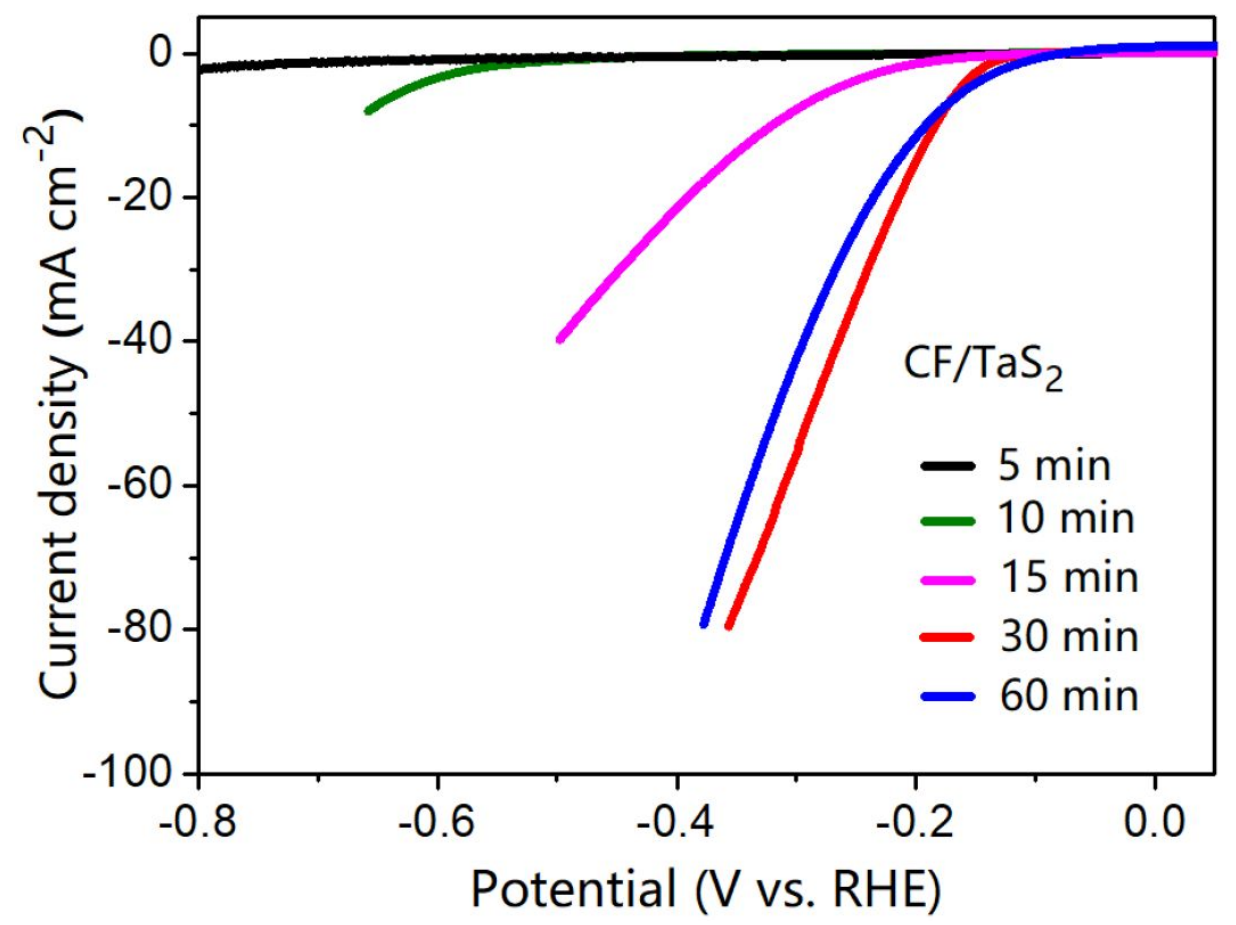

Figure S11. Polarization curves of $\mathrm{CF} / \mathrm{TaS}_{2}$ with different growth times from 5 min to $60 \mathrm{~min}$.

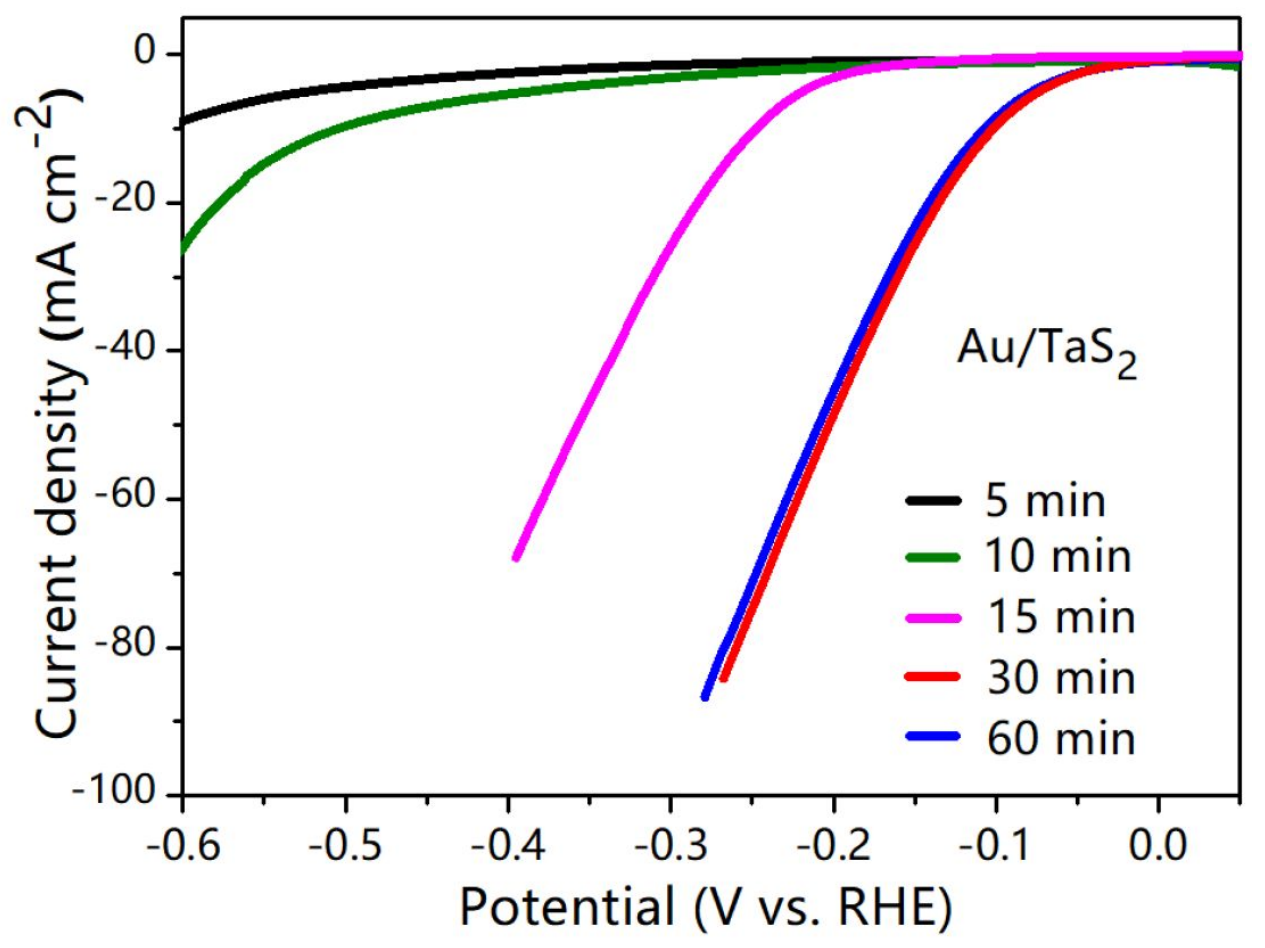


Figure S12. Polarization curves of $\mathrm{Au} / \mathrm{TaS}_{2}$ with different growth times from 5 min to $60 \mathrm{~min}$.

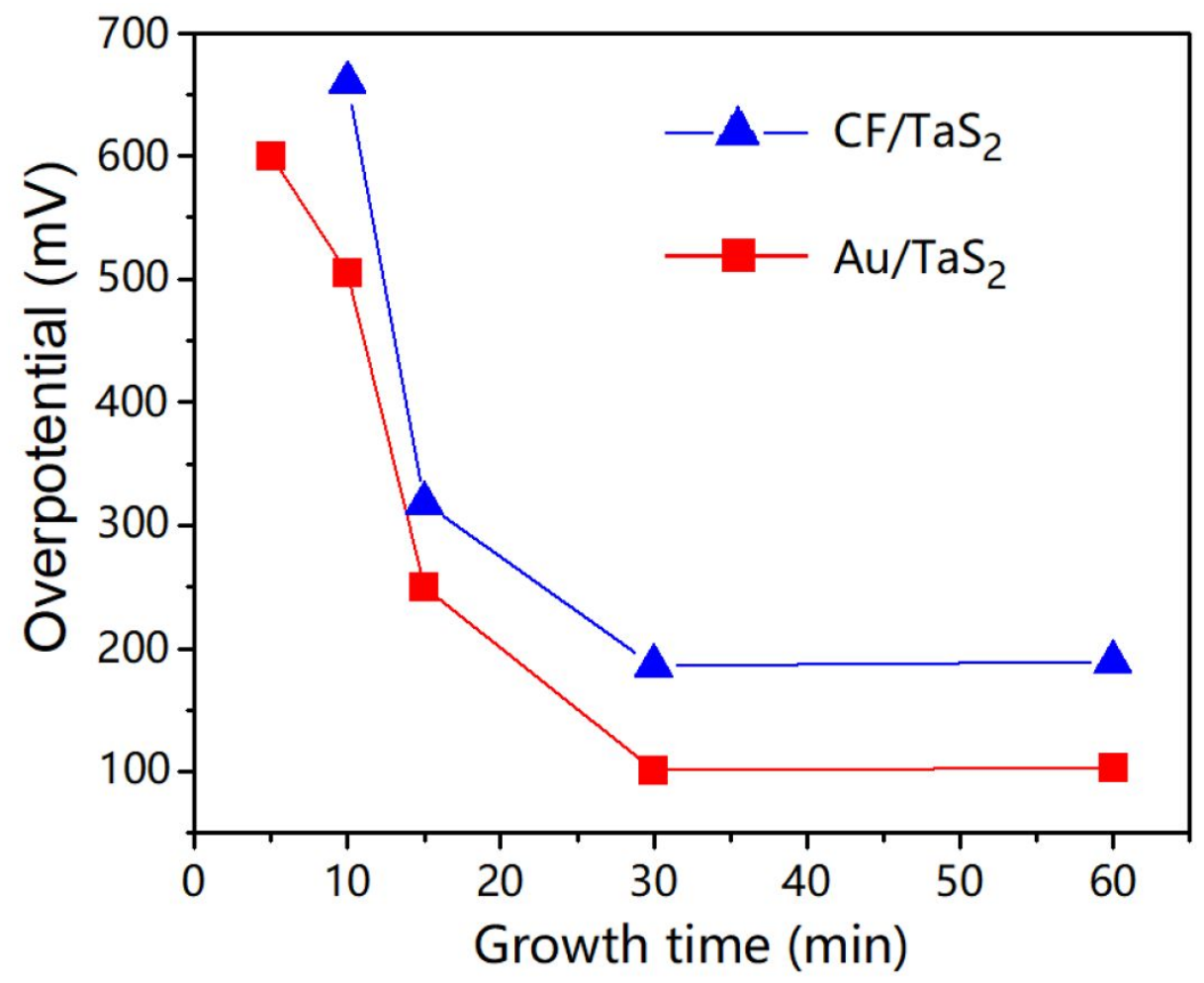

Figure S13. Overpotentials at $10 \mathrm{~mA} \mathrm{~cm}{ }^{-2}$ for $\mathrm{CF} / \mathrm{TaS}_{2}$ and $\mathrm{Au} / \mathrm{TaS}_{2}$ with different growth times from $5 \mathrm{~min}$ to $60 \mathrm{~min}$.
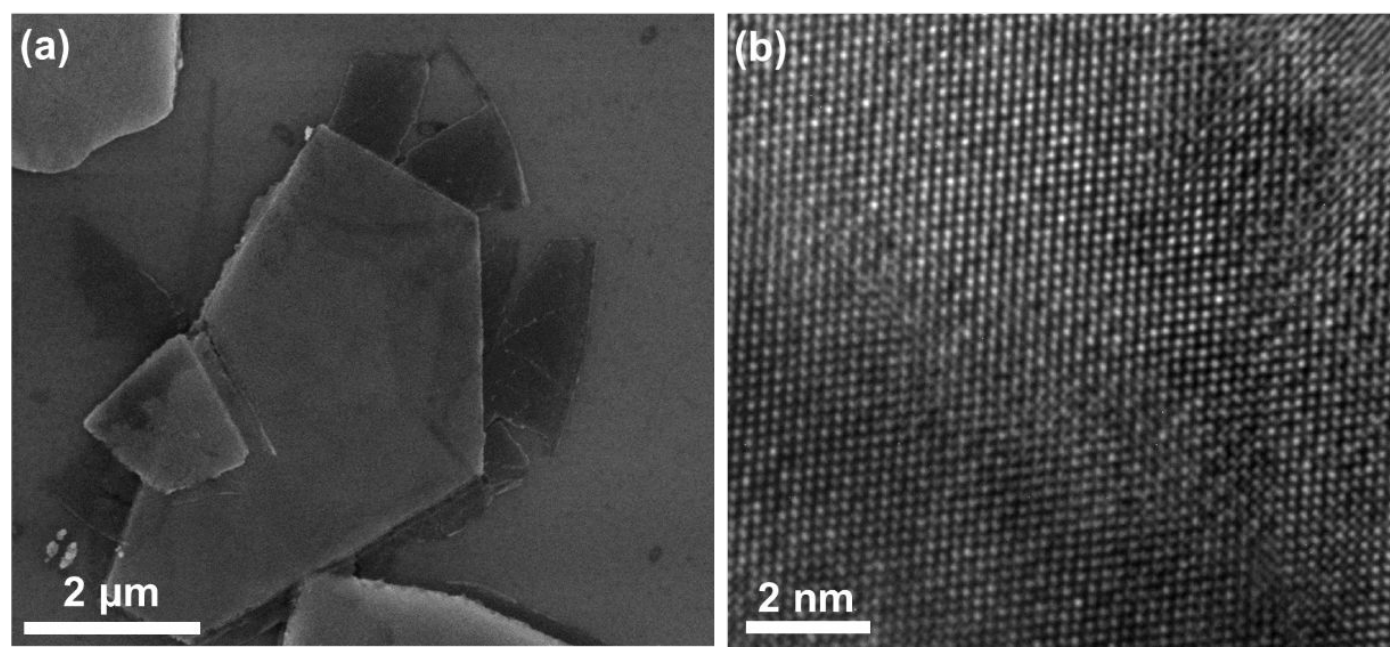

Figure S14. The SEM and HRTEM images of $\mathrm{Au} / \mathrm{TaS}_{2}$ catalyst obtained after stability test. 
Table S1. The HER activity comparisons of $\mathrm{TaS}_{2}$ and other TMDCs.

\begin{tabular}{|c|c|c|c|}
\hline Catalyst & $\begin{array}{l}\text { Overpotential at } \\
10 \mathrm{~mA} \mathrm{~cm}^{-2}(\mathrm{mV})\end{array}$ & $\begin{array}{c}\text { Tafel slopes }(\mathrm{mV} \\
\left.\operatorname{dec}^{-1}\right)\end{array}$ & References \\
\hline $\mathrm{TaS}_{2} / \mathrm{NbS}_{2}(2 \mathrm{H})$ & $60 / 50$ & $37 / 30$ & Nature energy 2017, 2, 17127 \\
\hline $\mathrm{Au} / \mathrm{TaS}_{2}(2 \mathrm{H})$ & $80-160$ & $45-49$ & $\begin{array}{c}\text { Nature communications, } \\
2017,5,958\end{array}$ \\
\hline $\mathrm{Au} / \mathrm{TaS}_{2}(1 \mathrm{~T})$ & 207 & 67 & $\begin{array}{l}\text { Advanced materials, 2018, } \\
1705916\end{array}$ \\
\hline $\mathrm{TaS}_{2}$ & 200 & 135 & $\begin{array}{c}\text { Advanced materials, } 2016, \\
28,8945-8949\end{array}$ \\
\hline $\mathrm{NbS}_{2}(2 \mathrm{H})$ & 500 & 74 & $\begin{array}{l}\text { Sustainable Energy Fuels, } \\
2018,2,96-102\end{array}$ \\
\hline $\mathrm{MoS}_{2}(1 \mathrm{~T})$ & 173 & 45 & $\begin{array}{l}\text { Advanced materials, 2018, } \\
1705509\end{array}$ \\
\hline $\mathrm{MoS}_{2}(1 \mathrm{~T})$ & 168 & 45 & $\begin{array}{c}\text { PNAS, 2013, 110, 19701- } \\
19706\end{array}$ \\
\hline $\mathrm{WS}_{2}(1 \mathrm{~T})$ & 140 & 70 & $\begin{array}{l}\text { Energy Environ. Sci, 2014, } 7 \text {, } \\
\text { 2608-2613 }\end{array}$ \\
\hline $\mathrm{WS}_{2}(1 \mathrm{~T})$ & 230 & 60 & $\begin{array}{l}\text { Nature Materials, } 2013,12 \text {, } \\
\qquad 850-855\end{array}$ \\
\hline $\mathrm{Au} / \mathrm{TaS}_{2}(2 \mathrm{H})$ & 101 & 53 & This work \\
\hline
\end{tabular}



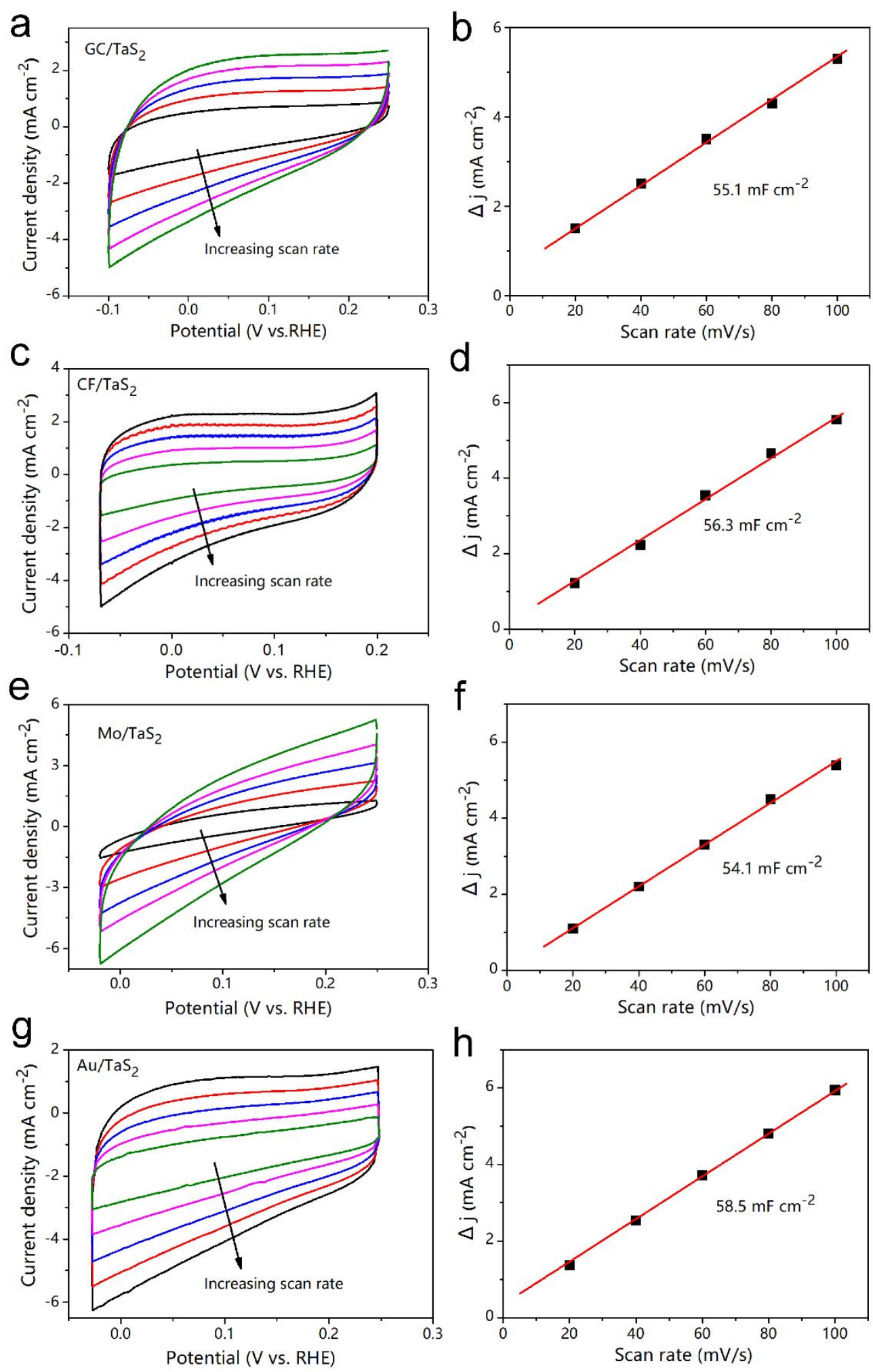

Figure S15. CV curves obtained with different scan rates from 20 to $100 \mathrm{mV} \mathrm{s}^{-1}$ in a $0.5 \mathrm{M} \mathrm{H}_{2} \mathrm{SO}_{4}$ solutions and a capacitive currents of $0.05 \mathrm{~V}$ against the scan rate and corresponding $C_{\mathrm{dl}}$ values estimated through linear fitting of the plots for different $\mathrm{TaS}_{2}$ samples. (a-b) GC/TaS 2 ; (c-d) CF/TaS $;$; (e-f) Mo/TaS 2 ; (g-h) $\mathrm{Au} / \mathrm{TaS}_{2}$. 


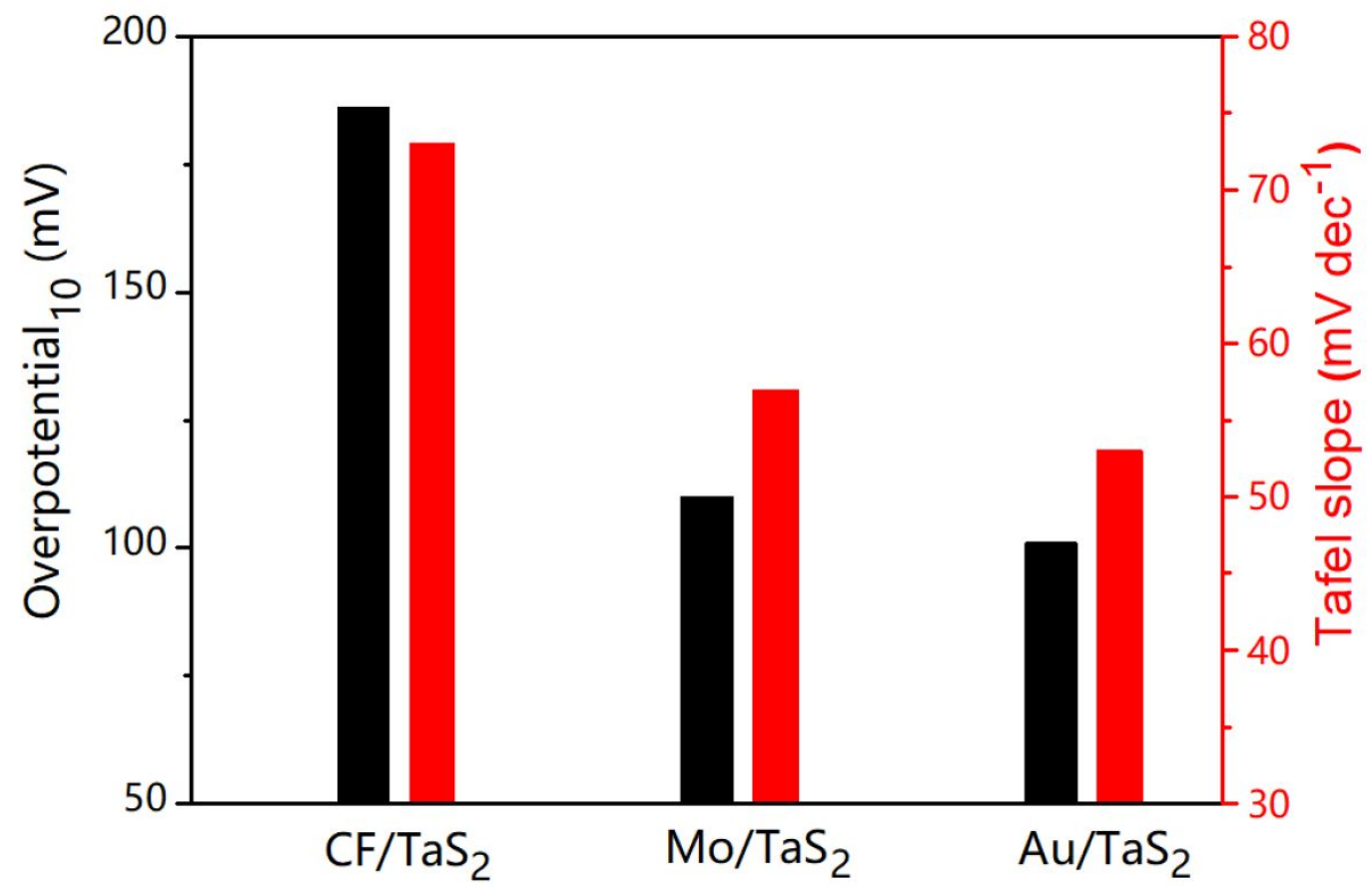

Figure S16. The Overpotentials (left y-axis) at a current density of $10 \mathrm{~mA} \mathrm{~cm}^{-2}$ and Tafel slopes (right y-axis) of $\mathrm{CF} / \mathrm{TaS}_{2}, \mathrm{Mo} / \mathrm{TaS}_{2}$, and $\mathrm{Au} / \mathrm{TaS}_{2}$. 


\section{References:}

(1) Liu, Y. Y.; Wu, J. J.; Hackenberg, K. P.; Zhang, J.; Wang, Y. M.; Yang, Y. C.; Keyshar, K.; Gu, J.; Ogitsu, T.; Vajtai, R.; Lou, J.; Ajayan, P. M.; Wood, B. C.; Yakobson, B. I. Self-Optimizing, Highly Surface-Active Layered Metal Dichalcogenide Catalysts for Hydrogen Evolution. Nat. Energy 2017, 2, 17127.

(2) Shi, J.; Wang, X.; Zhang, S.; Xiao, L.; Huan, Y.; Gong, Y.; Zhang, Z.; Li, Y.; Zhou, X.; Hong, M.; Fang, Q.; Zhang, Q.; Liu, X.; Gu, L.; Liu, Z.; Zhang, Y. TwoDimensional Metallic Tantalum Disulfide as a Hydrogen Evolution Catalyst. Nat. Commun. 2017, 8, 958.

(3) Huan, Y.; Shi, J.; Zou, X.; Gong, Y.; Zhang, Z.; Li, M.; Zhao, L.; Xu, R.; Jiang, S.; Zhou, X.; Hong, M.; Xie, C.; Li, H.; Lang, X.; Zhang, Q.; Gu, L.; Yan, X.; Zhang, Y. Vertical $1 \mathrm{~T}-\mathrm{TaS}_{2}$ Synthesis on Nanoporous Gold for High-Performance Electrocatalytic Applications. Adv. Mater. 2018, 30, e1705916.

(4) Li, H.; Tan, Y. W.; Liu, P.; Guo, C. G.; Luo, M.; Han, J. H.; Lin, T. Q.; Huang, F. Q.; Chen, M. W. Atomic-Sized Pores Enhanced Electrocatalysis of $\mathrm{TaS}_{2}$ Nanosheets for Hydrogen Evolution. Adv. Mater. 2016, 28, 8945-8949.

(5) Gopalakrishnan, D.; Lee, A.; Thangavel, N. K.; Arava, L. M. R. Facile Synthesis of Electrocatalytically Active $\mathrm{NbS}_{2}$ Nanoflakes for an Enhanced Hydrogen Evolution Reaction (HER). Sustain. Energ. Fuels 2018, 2, 96-102.

(6) Tan, C. L.; Luo, Z. M.; Chaturvedi, A.; Cai, Y. Q.; Du, Y. H.; Gong, Y.; Huang, Y.; Lai, Z. C.; Zhang, X.; Zheng, L. R.; Qi, X. Y.; Goh, M. H.; Wang, J.; Han, S. K.; Wu, X. J.; Gu, L.; Kloc, C.; Zhang, H. Preparation of High-Percentage 1T-Phase Transition Metal Dichalcogenide Nanodots for Electrochemical Hydrogen Evolution. Adv. Mater. 2018, 30,1705509

(7) Wang, H. T.; Lu, Z. Y.; Xu, S. C.; Kong, D. S.; Cha, J. J.; Zheng, G. Y.; Hsu, P. C.; Yan, K.; Bradshaw, D.; Prinz, F. B.; Cui, Y. Electrochemical Tuning of Vertically Aligned $\mathrm{MoS}_{2}$ Nanofilms and Its Application in Improving Hydrogen Evolution Reaction. P. Natl. Acad. Sci. USA 2013, 110, 19701-19706.

(8) Lukowski, M. A.; Daniel, A. S.; English, C. R.; Meng, F.; Forticaux, A.; Hamers, R. J.; Jin, S. Highly Active Hydrogen Evolution Catalysis from Metallic $\mathrm{WS}_{2}$ Nanosheets. Energ. Environ. Sci. 2014, 7, 2608-2613.

(9) Voiry, D.; Yamaguchi, H.; Li, J. W.; Silva, R.; Alves, D. C. B.; Fujita, T.; Chen, M. W.; Asefa, T.; Shenoy, V. B.; Eda, G.; Chhowalla, M. Enhanced Catalytic Activity in Strained Chemically Exfoliated $\mathrm{WS}_{2}$ Nanosheets for Hydrogen Evolution. Nat. Mater. 2013, 12, 850-855. 